\title{
An approach to compare the quality of cancellous bone from the femoral necks of healthy and osteoporotic patients through compression testing and microcomputed tomography imaging
}

\author{
Anthony Ciarallo*, Jake Barralet, Michael Tanzer, Richard Kremer
}

\begin{abstract}
It is estimated that osteoporosis is responsible for about 300000 hip fractures per year in the United States. Effective prevention of these fractures has been demonstrated using bisphosphonates. However, their mechanism of action has not been elucidated. Furthermore, the precise effect of bisphosphonates on the femoral neck and surrounding areas has never been studied. We are interested in establishing a protocol to analyze the bone quality of proximal femurs from patients treated with bisphosphonates. Following hip replacement surgery, the aim is to determine whether imaging and compression testing of cancellous bone from the discarded femoral necks can accurately assess the bone's microarchitectural and biomechanical properties, respectively. To validate the technique, it was first tested on an untreated population. A bone biopsy trephine was used to extract cylindrical cores of trabecular bone from the centre of femoral necks. Densitometry, microcomputed tomography, and compression testing were used to assess the quality of bone in these samples. The compressive strength was found to be directly proportional to the modulus (i.e. stiffness) of the samples, thus reproducing previous findings. The relative porosity and, to a lesser extent, the bone mineral density were capable of predicting the quality of cancellous bone. In conclusion, a protocol to analyze the bone quality in human femoral necks using $\mu \mathrm{CT}$ and biomechanical compression testing was successfully established. It will be applied in a clinical setting to analyze bones from bisphosphonate-treated patients following total hip replacement.
\end{abstract}

KEYWORDS: microcomputed tomography, biomechanics, bisphosphonates, femoral neck, cancellous, osteoporosis, hip fracture

\section{INTRODUCTION}

Osteoporosis is defined by the World Health Organization (WHO) as having a bone mineral density (BMD) of more than 2.5 standard deviations below the norm for healthy young adults (1). BMD, measured by dual-energy X-ray absorptiometry, is used as a bone mass index to predict the risk of fracture (1).

Osteoporotic bones are porous and fragile, thus

*To whom correspondence should be addressed: Anthony Ciarallo anthony.ciarallo@mail.mcgill.ca making them more susceptible to fracture than healthy bones. In order to maintain a healthy skeleton, a steadystate of bone remodeling occurs in adults in which new bone is generated at the same rate that old bone is removed. The remodeling process is important for maintaining bone strength because it adapts the structure of bone to mechanical loads (2). In osteoporosis, the rate of bone resorption exceeds that of the formation, resulting in a progressive deterioration of bone mass and microarchitecture (2).

There are the two kinds of bone that comprise the 
skeleton are compact and cancellous (i.e. trabecular). Compact bone predominates in the appendicular skeleton and is designed to resist bending forces. On the other hand, cancellous bone is concentrated in the axial skeleton and is structured to resist compressive forces, including gravity. Since it is lighter and more porous than compact bone, cancellous bone provides more surface area for bone remodeling and is more metabolically active (2). Consequently, cancellous bone is more affected in osteoporosis (3).

One class of drugs aimed at preventing bone loss is bisphosphonates. Bisphosphonates, including alendronate and risedronate, are antiresorptive agents which are stable analogues of inorganic pyrophosphate and have a high affinity for hydroxyapatite crystals. They bind selectively to mineralized surfaces of bone to disrupt degradative osteoclast activity and promote apoptosis of mature osteoclasts (4). Bisphosphonates are also thought to prolong the life of osteoblasts by inhibiting apoptosis (5).

Alendronate and risedronate are highly effective in decreasing the risk of vertebral and hip fractures (6-8). But, it was also demonstrated that the observed increase in BMD in bisphosphonate-treated patients does not entirely account for the reduction in fracture risk (6). Therefore, the mechanism of action of bisphosphonates has not yet been elucidated. In fact, it is hypothesized that bisphosphonates may act by increasing bone mass, improving trabecular microarchitecture, and/or increasing secondary mineralization of the calcified matrix (9). Recently, more emphasis has been placed on the concept of "bone quality" which encompasses the microarchitectural and biomechanical properties of bone $(10,11)$.

It is estimated that osteoporosis is responsible for about 300000 hip fractures per year in the United States (12). Recent studies have shown that hip-fractured patients rapidly lose bone density within 6 months after fracture, possibly because of the absence of weight bearing for 6 weeks post-operatively and the fact that peri-prosthetic bone loss rapidly occurs following hip arthroplasty (13). Since there is no standard of care for the prevention and the treatment of osteoporosis in these patients, our interest is to determine the best approach to prevent bone loss in this group. As the effect of bisphosphonates on the femoral neck and surrounding areas has not yet been assessed, we will analyze the bone quality of proximal femurs from treated patients that are discarded after hip replacement surgery. Prior to undertake these clinical trials, however, protocols must be established in an untreated population in order to properly assess the quality of bone.

This project aims to determine whether imaging and compression testing of cancellous bone from the femoral neck can accurately assess the bone's microarchitectural and biomechanical properties, respectively, and if together they will serve as a good index of bone quality.

\section{MATERIALS AND METHODS}

Twenty-two bovine (calf) femurs were obtained from a butcher (Boucherie Charcuterie Rivière des Prairies, Montreal, QC). Nineteen human proximal femurs were collected by Dr. Michael Tanzer (Montreal General Hospital, Montreal, QC) and Dr. William Fisher (Montreal General Hospital, Montreal, QC) from total hip replacement orthopedic surgeries. Patients signed consent forms for the donation of their tissue. All samples were wrapped in gauze and soaked in saline to prevent drying. The specimens were frozen at $-30^{\circ} \mathrm{C}$ until bone core extraction (14).

Prior to sample preparation, the femurs were thawed at $4^{\circ} \mathrm{C}$ overnight. A Rochester Bone Biopsy Trephine (Item \#87400-000, Gauthier Medical Inc., Rochester, MN) $7.5 \mathrm{~mm}$ in diameter was used to extract cylindrical cores of trabecular bone from the centre of the femoral necks. Both ends of the cores were cut at right angles to the long axis in order to minimize shearing during biomechanical compression testing (15). The dimensions of the trabecular bone cores were $7.5 \mathrm{~mm}$ in diameter by $14 \mathrm{~mm}$ in length, thereby maintaining a valid aspect ratio between 1.5 and 2 (16). The cores were placed in $70 \%$ ethanol prior to testing (17).

The trabecular bone cores were scanned using a Lunar PIXImus densitometer (Lunar Corp, Madison, WI) at the Bone Centre (McGill University, Montreal, $\mathrm{QC})$ to measure the bone mineral density (BMD) for a region of interest inside the specimen $(11.3 \mathrm{~mm} \times 5 \mathrm{~mm})$. They were subsequently scanned with a microcomputed tomography $(\mu \mathrm{CT})$ instrument (Model 1072, Skyscan, Aartselaar, Belgium) at the Bone Centre (McGill University, Montreal, QC). The cross-sections along the specimen axis were reconstructed using ConeBeam Reconstruction software (SkyScan), with a distance between each cross-section of $18.25 \mu \mathrm{m}$. The CT-Analyser software (SkyScan) was used to analyze the trabecular bone of the sample using segmentation method.

The bone cores were then subjected to the compression testing. Specimens were mounted on the testing machine (Instron 5569, Buckinghamshire, UK) so that the long axes of the cylinders were perpendicular to the lower anvil. A compressive force was then applied to the upper surface of the samples using a $50 \mathrm{kN}$ load cell at a constant crosshead displacement rate of $1 \mathrm{~mm} / \mathrm{min}$ until failure occurred. The compressive load and the sample length were recorded at 0.1 second intervals during compression. Using this 
data, the Stress (Force/Area) and Strain (Length/Original Length) were calculated and plotted against one another. The Elastic Modulus, an indication of the extent of compressive deformation in a specimen for a given load, was obtained from the linear portion of the Stress versus Strain curve (15).

\section{RESULTS}

Initially, it was imperative to demonstrate that the protocol used to retrieve cylindrical cores of cancellous bone would provide valid samples for biomechanical testing. Twenty two cores of cancellous bone were extracted from the femoral neck of calf femurs and subjected to compression testing. The compressive strength and the elastic modulus were calculated for each sample and plotted against each other. It should be noted that the compressive strength is a measure of the stress within a sample immediately before the yielding point, while the elastic modulus is a measure of the rigidity of a sample. In concordance with previous findings, the compressive strength correlates strongly to the modulus $(r=0.98)$ in the calf femur samples (see Figure 1) (18). Furthermore, these parameters fall within the range of published bovine values (19).

Upon validating our technique, the same procedure was applied to the human femoral neck samples obtained from hip replacement surgeries. Nineteen human samples were obtained and tested. Comparable to the bovine samples, the compressive strength and the modulus of the human samples are strongly correlated $(r=0.99)$, as demonstrated in Figure 2. Moreover, the values for these parameters fall within the range of published human values for the femoral neck (20).

Prior to subjecting the human cancellous samples to biomechanical testing, they were scanned using three dimensional $\mu \mathrm{CT}$ and densitometry. As demonstrated in Figure 3, the osteoporotic bone appears much more porous than the unaffected bone. The compressive strength was plotted against the trabecular thickness, number and separation, as shown in Figure 4. The latter parameters failed to show any statistically significant relationship to the biomechanics of bone. The trabecular thickness and separation remained fairly constant despite the strength of the bone, whereas the number of trabeculae appeared to increase with the compressive strength although not well. Needless to say, the trabecular data proved to be poor indicators of bone quality.

Further investigation of the imaging data revealed that the relative porosity, calculated as the complement of the percent bone volume (i.e. 1 - \% bone volume), and the BMD were the best predictors of bone quality. In fact, the relative porosity and the BMD correlated strongly to one another (see Figure $5, \mathrm{r}=0.97$ ). Upon analyzing the data, the compressive strength and the modulus were shown to vary exponentially with relative porosity and the BMD. That is to say, the strength and the modulus of the samples increased at a faster rate as the relative porosity decreased or the BMD increased. Thus, the latter were plotted against the natural logarithms of the compressive strength and the modulus for each bone sample (see Figures 6-9). The relative porosity and the BMD correlated equally well to the compressive strength and the modulus ( $\mathrm{Ln}$ (compressive strength) vs. relative porosity, $\mathrm{r}=-0.90 ; \mathrm{Ln}$ (modulus) vs. relative porosity, $\mathrm{r}=-0.88$; $\mathrm{Ln}$ (compressive strength) vs. BMD, $\mathrm{r}=0.94$; Ln(modulus) vs. BMD, $\mathrm{r}=$ 0.92).

\section{DISCUSSION}

The assessment of bone quality is of the utmost

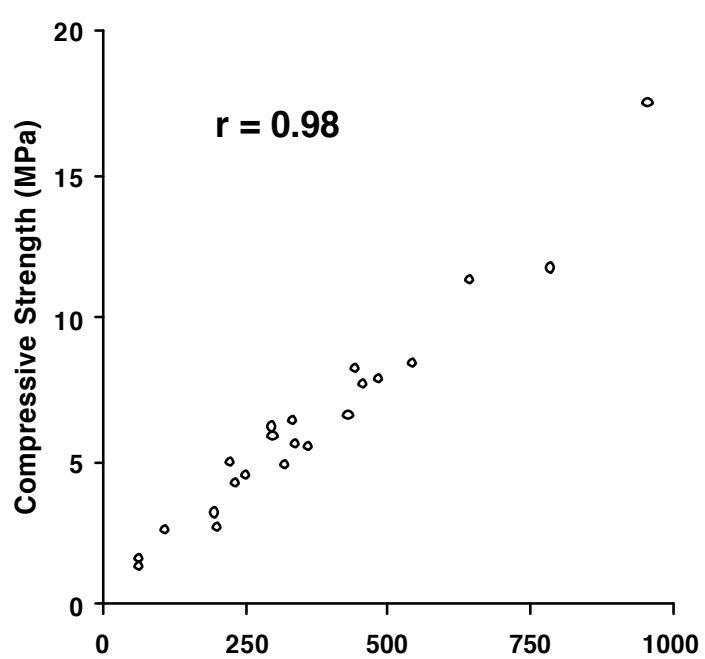

Figure 1: Compressive strength versus Modulus in cancellous bone from calf femoral necks. Both parameters are measured in MegaPascals (MPa). Correlation coefficient, $r=0.98$

importance in osteoporotic patients as it correlates well to the risk of fracture (1) and indicates when it is appropriate to initiate the proper preventative measures (6-8). Bisphosphonates have proven to be quite successful in deterring the progression of osteoporosis (6). However, their precise mechanism of action has yet to be determined. Recently, there have been trials that have studied the effect of risedronate in osteoporotic patients using $\mu \mathrm{CT}$ (11). These studies have indirectly assessed the drug's effect on bone quality using a remote site (i.e. the iliac crest) rather than the site of interest (e.g. vertebrae, femoral neck). Understandably, the analysis of the iliac crest is convenient because it is easily accessible from the surface and poses minimal risk to the patient. There has been, however, no evidence to indicate whether any correlation exists 


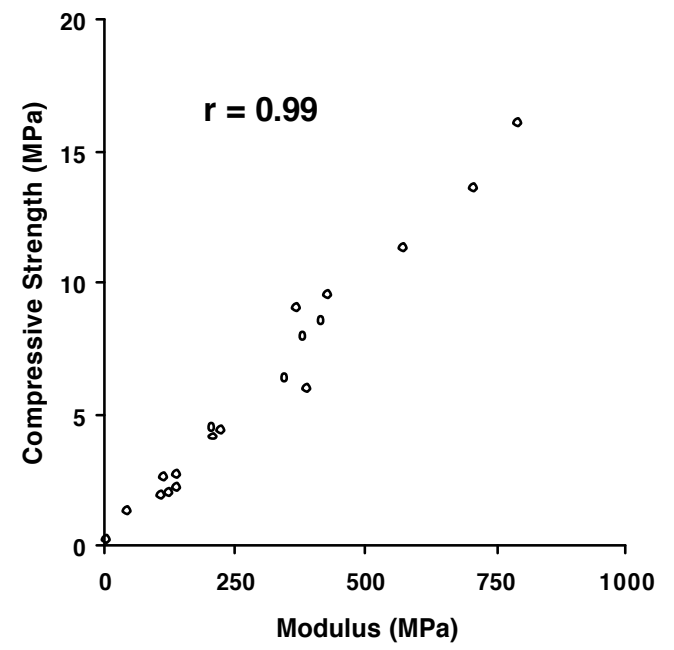

Figure 2: Compressive strength versus Modulus in cancellous bone from human femoral necks. Both parameters are measured in MegaPascals (MPa). Correlation coefficient, $\mathrm{r}=0.99$.

between the microarchitecture at the iliac crest and the site of interest. To our knowledge, the effects of bisphosphonates on bone quality have not yet been studied in humans at sites than at the iliac crest.

Prior to embarking on a hip fracture study, we wanted to establish reliable and reproducible procedures for biomechanical testing to ensure that the data were valid and representative of bone quality. Intuitively, bone quality should diminish as the bone becomes more porous and/or less dense with mineral. Indeed, we found the relative porosity and the BMD to be equally strong indicators of bone quality as both of these parameters correlate strongly to the biomechanical properties of bone, though in opposite fashion (see Figures 6-9).

A potential limitation to this experiment is the inherent design flaw that results from the extraction of trabecular bone. As the cylindrical core of bone is removed from the sample, the cross struts from the trabeculae along the periphery are severed. This unavoidably compromises structural integrity, especially since the compact bone significantly contributes to the overall strength of the femur (21). Hence, the biomechanical studies will not be entirely representative of the properties of trabeculae within the bone. However, taking into consideration that all the samples are prepared identically before testing, the data analysis remains valid.

\section{CONCLUSION}

We have successfully established a protocol that will be used in a trial setting to analyze the bone quality in human femoral necks using $\mu \mathrm{CT}$ and biomechanical compression testing. Using cores of cancellous bone from human femoral necks, the relative porosity and the BMD were shown to be excellent predictive markers of fracture resistance. As this trial evolves in its early stages, additional samples collected from hip replacements will be tested and the data compiled to construct a reference database for comparison to bisphosphonate treatment cohorts.

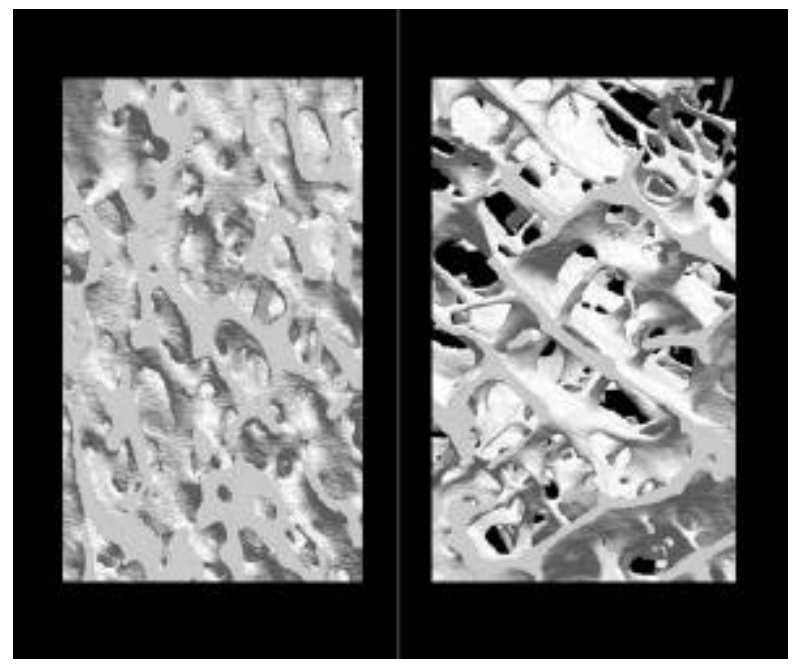

Figure 3: Computer-generated three dimensional images of unaffected bone (left panel) and osteoporotic bone (right panel) from $\mu \mathrm{CT}$ scans.

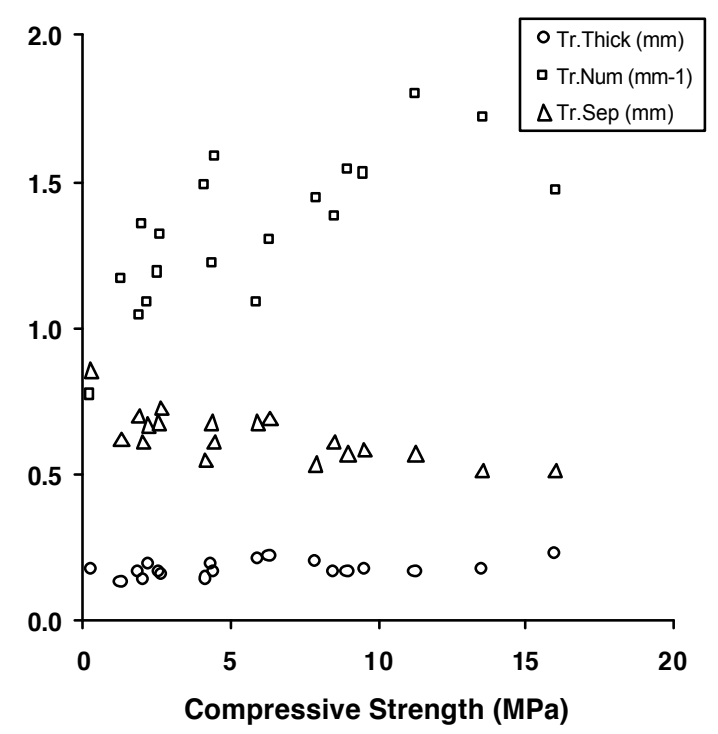

Figure 4: Trabecular thickness (Tr.Thick, circles), number (Tr.Num, squares), and separation (Tr.Sep, triangles) plotted against Compressive Strength in MegaPascals (MPa). Trabecular thickness and separation are measured in millimeters $(\mathrm{mm})$ and trabecular number is measured as the quantity of trabeculae per millimeter $\left(\mathrm{mm}^{-1}\right)$. 


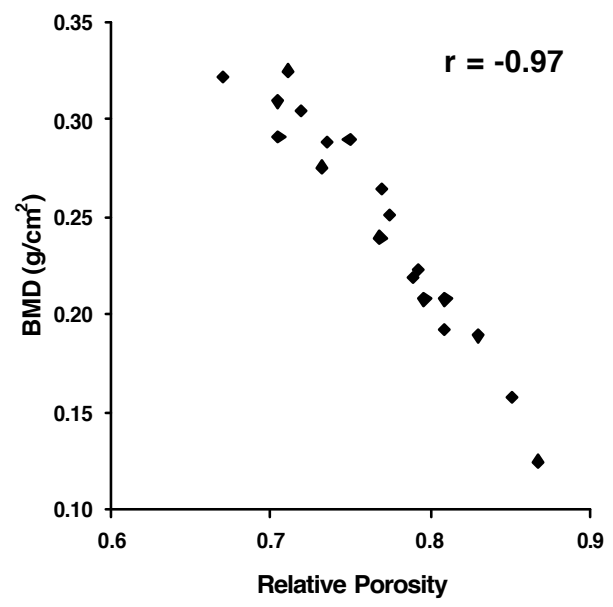

Figure 5: BMD plotted against Relative Porosity. Correlation coefficient, $r=-0.97$.

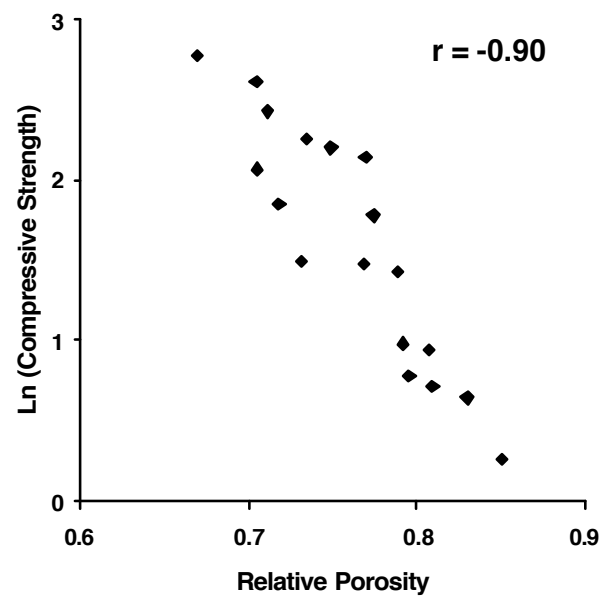

Figure 6: The natural logarithm of Compressive Strength plotted against Relative Porosity. Correlation coefficient, $r=-0.90$.

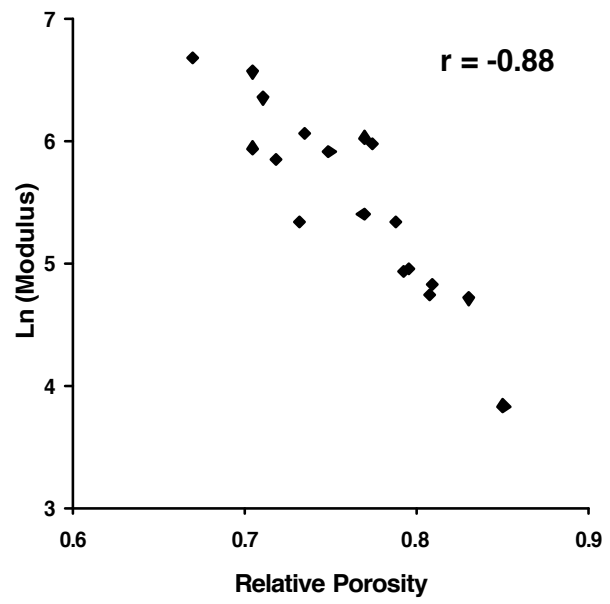

Figure 7: The natural logarithm of Modulus plotted against Relative Porosity. Correlation coefficient, $\mathrm{r}=-0.88$.

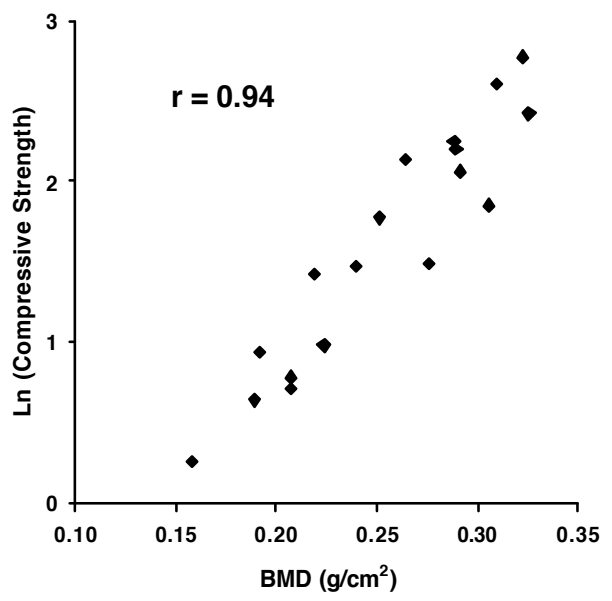

Figure 8: The natural logarithm of Compressive Strength plotted against BMD. Correlation coefficient, $\mathrm{r}=0.94$.

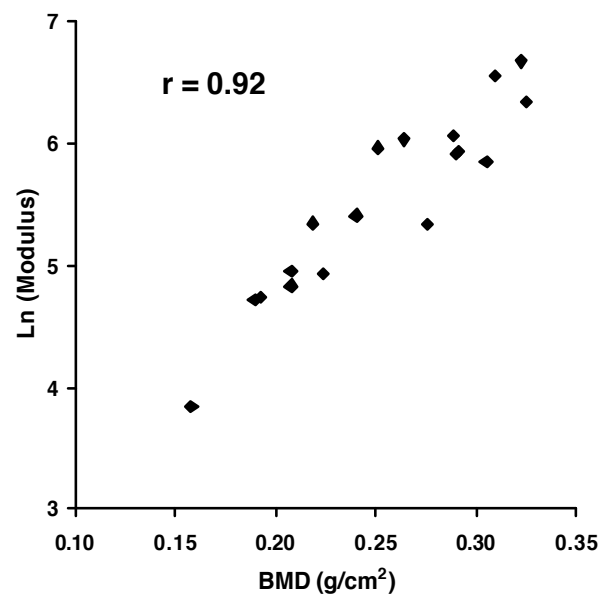

Figure 9: The natural logarithm of Modulus plotted against BMD. Correlation coefficient, $r=0.92$.

\section{REFERENCES}

1. WHO, Assessment of fracture risk and its application to screening for postmenopausal osteoporosis, in Report of a WHO study group. WHO technical report series no. 8430. 1994, World Health Organization: Geneva, Switzerland.

2. Boonen, S., et al., Preventing osteoporotic fractures with antiresorptive therapy: implications of microarchitectural changes. J Intern Med, 2004; 255(1): p. 1-12.

3. Seeman, E., Invited Review: Pathogenesis of osteoporosis. J Appl Physiol, 2003; 95(5): p. 2142-51.

4. Russell, R.G., et al., The pharmacology of bisphosphonates and new insights into their mechanisms of action. J Bone Miner Res, 1999; 14(Suppl 2): p. 53-65.

5. Manolagas, S.C., Birth and death of bone cells: basic regulatory mechanisms and implications for the pathogenesis and treatment of osteoporosis. Endocr Rev, 2000; 21(2): p. 115-37.

6. Black, D.M., et al., Randomised trial of effect of alendronate on risk of fracture in women with existing vertebral fractures. 
Fracture Intervention Trial Research Group. Lancet, 1996; 348(9041): p. 1535-41.

7. Ensrud, K.E., et al., Treatment with alendronate prevents fractures in women at highest risk: results from the Fracture Intervention Trial. Arch Intern Med, 1997; 157(22): p. 2617-24.

8. Harris, S.T., et al., Effects of risedronate treatment on vertebral and nonvertebral fractures in women with postmenopausal osteoporosis: a randomized controlled trial. Vertebral Efficacy With Risedronate Therapy (VERT) Study Group. Jama, 1999; 282(14): p. 1344-52.

9. Day, J.S., et al., Bisphosphonate treatment affects trabecular bone apparent modulus through micro-architecture rather than matrix properties. J Orthop Res, 2004; 22(3): p. 465-71.

10. Osteoporosis prevention, diagnosis, and therapy. Jama, 2001; 285(6): p. 785-95.

11. Borah, B., et al., Risedronate preserves bone architecture in postmenopausal women with osteoporosis as measured by three-dimensional microcomputed tomography. Bone, 2004; 34(4): p. 736-46.

12. Passi, N. and A. Gefen, Trabecular bone contributes to strength of the proximal femur under mediolateral impact in the avian. $\mathrm{J}$ Biomech Eng, 2005; 127(1): p. 198-203.

13. Rosenthall, L., J.D. Bobyn, and M. Tanzer, Bone densitometry: influence of prosthetic design and hydroxyapatite coating on regional adaptive bone remodeling. Int Orthop, 1999; 23(6): p. $325-9$.
14. Panjabi, M.M., et al., Biomechanical time-tolerance of fresh cadaveric human spine specimens. J Orthop Res, 1985; 3(3): p. 292-300.

15. Currey, J.D., The mechanical properties of bone. Clin Orthop Relat Res, 1970; 73: p. 209-31.

16. Price, W., Factors influencing concrete strength. J Am Con Inst, 1995; 31: p. 417-33.

17. Linde, F. and H.C. Sorensen, The effect of different storage methods on the mechanical properties of trabecular bone. $\mathrm{J}$ Biomech, 1993; 26(10): p. 1249-52.

18. Hodgskinson, R. and J.D. Currey, Effects of structural variation on Young's modulus of non-human cancellous bone. Proc Inst Mech Eng (H), 1990; 204(1): p. 43-52.

19. Hodgskinson, R. and J.D. Currey, The effect of variation in structure on the Young's modulus of cancellous bone: a comparison of human and non-human material. Proc Inst Mech Eng (H), 1990; 204(2): p. 115-21.

20. Martens, M., et al., The mechanical characteristics of cancellous bone at the upper femoral region. J Biomech, 1983; 16(12): p. 971-83.

21. Lotz, J.C., E.J. Cheal, and W.C. Hayes, Stress distributions within the proximal femur during gait and falls: implications for osteoporotic fracture. Osteoporos Int, 1995; 5(4): p. 252-61.

Anthony Ciarallo graduated from McGill in 2002 with a BSc in Physiology and in 2005 with an MSc in Human Genetics. His graduate research at the Shriners Hospital for Children aimed at elucidating the signaling pathways involved in the regulation of embryonic endochondral bone development. The goal of the research was to identify potential treatments for congenital skeletal dysplasias such as achondroplasia. 